\title{
ASSIMILAÇÃO DE CO2 DE GENÓTIPOS MODERNOS E TRADICIONAIS DE ARROZ SUBMETIDOS AO ESTRESSE HÍDRICO
}

\author{
Rogério Lorençoni ${ }^{1}$ \\ ${ }^{1}$ Escola Superior de Agricultura "Luiz de Queiroz" - E-mail: rogeriolorenconi@gmail.com
}

\section{RESUMO}

O objetivo deste trabalho foi avaliar a assimilação de CO2(A) de dez genótipos de arroz, três pertencentes ao grupo moderno (BRS-Cirrad, BRS-Curinga e BRS-Primavera) e sete ao grupo tradicional (Douradão, Agulha Ligeiro, Bolinha, Batatais, Três Meses Branco, Beira Campo e De Morro) em resposta a alteração da disponibilidade hídrica do solo. As plantas foram cultivadas em vasos, mantidas dentro de casa de vegetação sem restrição hídrica até apresentarem 50\% de seus perfilhos no estádio R2, sendo posteriormente transferidas para uma câmara de crescimento com ambiente controlado (Fitotron), onde foram submetidas a duas condições de disponibilidade hídrica, uma próximo à capacidade máxima de retenção de água do solo, e outra em uma tensão de $40 \pm 2 \mathrm{kPa}$. Na condição de maior disponibilidade hídrica observa-se a superioridade dos genótipos modernos em relação aos tradicionais. Ao compararmos os genótipos nas duas condições de disponibilidade hídrica, observa-se que os modernos apresentam as maiores reduções de (A) em relação aos tradicionais sob menor disponibilidade hídrica, demonstrando maior susceptibilidade ao déficit hídrico, ao passo que os tradicionais, apesar de apresentarem maior tolerância nas condições de menor disponibilidade hídrica, apresentam menores (A) sob condições bem irrigadas.

Palavras-chave: Oryza sativa, deficit hídrico, tolerância à seca, ajustamento estomático

\section{CO2 ASSIMILATION OF MODERN AND TRADITIONAL RICE GENOTYPES SUBJECTED TO WATER STRESS}

\begin{abstract}
The objective of this study was evaluate the $\mathrm{CO}_{2}$ assimilation $(A)$ in the ten rice genotypes, three belonging to the modern group (BRS-Cirrad, BRS-Primavera, BRS-Curinga) and seven the traditional group (Douradão, AgulhaLigeiro, Bolinha, Batatais, TrêsMesesBranco, Beira Campo e De Morro) in response to changes in water availability in the soil. Plants were grown in pots, maintained in a greenhouse without water restriction to exhibit $50 \%$ of their tillers in the R2 stage, later being transferred to a growth chamber with controlled environment (Fitotron), where they
\end{abstract}


were subjected to two conditions of availability water, one near the maximum water holding capacity of the soil, and the other in tension of $40 \pm 2 \mathrm{kPa}$. As the largest water availability shows the superiority of modern genotypes compared to traditional. When comparing the genotypes in both water availability conditions, it is observed that the modern present the higher reductions of (A) compared to traditional in lower hydric availability, demonstrated a higher susceptibility to water deficit, whereas traditional, despite showing superior tolerance under the conditions of lower water availability, have lower $(A)$ under well-watered conditions.

Keywords: Oryza sativa, water stress, drought tolerance, stomatal adjustment

\section{INTRODUÇÃO}

Cultivares modernos de arroz de terras altas, quando cultivados em áreas favorecidas quanto à distribuição de chuvas, como no Centro-Norte do MT, podem apresentar produtividades superiores a $4 \mathrm{t} \mathrm{ha}^{-1}$, no entanto, a média nacional de produtividade é de apenas 1,8 $\mathrm{t} \mathrm{ha}^{-1}$ (PINHEIRO, 2003). Essa produtividade média reduzida tem sido atribuída principalmente à irregularidade ocorrida na distribuição das chuvas, ocasionando pequenos períodos de escassez conhecidos como veranicos (ARF et al., 2000; CRUSCIOL et al., 2003a,b).

Plantas de arroz submetidas as condições de alta demanda evapotranspirativa e baixa disponibilidade hídrica que ocorrem durante o veranico, tornam-se dependentes do mecanismo de ajustamento estomático foliar, o qual, tende a minimizar a perde de água por transpiração ao mesmo tempo em que tenta manter a captura de carbono $\left(\mathrm{CO}_{2}\right)$ utilizado na assimilação, necessário para o crescimento e desenvolvimento das plantas. De acordo com Yoshida (1975) e Murchie et al., (1999),a maior parte do carbono armazenado nos grãos de arroz, tem sua origem na assimilação de $\mathrm{CO}_{2}$ ocorrida nas folhas bandeira. Assim, fatores que venham a reduzir a taxa de assimilação de $\mathrm{CO}_{2}$ da folha bandeira durante período reprodutivo podem limitar potencialmente o rendimento de grãos da cultura (DINGKUHN et al., 1989).

Neste contexto, a utilização de cultivares melhoradas capazes de apresentar maiores taxas de assimilação sob condições de déficit hídrico, torna-se uma estratégia promissora para minimizar os efeitos do veranico sobre a produtividade do arroz de terras altas.

O cruzamento entre genótipos de alta produtividade, porém, susceptíveis ao déficit 
hídrico, com genótipos de menor potencial produtivo, mas tolerantes ao déficit hídrico, tem gerado genótipos capazes de aliar elevado potencial produtivo mesmo em ambientes com baixa disponibilidade hídrica (VENUPRASAD et al., 2007; KUMAR et al., 2008).

A identificação de genótipos promissores nos cruzamentos dos programas de melhoramento de tolerância a seca, pode ser otimizada através da avaliação e comparação de cultivares desenvolvidos pelos programas de melhoramento moderno, capazes de apresentar elevados potenciais produtivos (RANGEL et al., 2011; RABELO et al., 2006), bem como das variedades tradicionais ou crioulas, capazes de apresentar elevada tolerância ao déficit hídrico (AREIAS et al., 2006).

Dessa maneira, este trabalho tem como objetivo avaliar e comparara taxa de assimilação de $\mathrm{CO}_{2}$ da folha bandeira de genótipos tradicionais e modernos de arroz cultivados em terras altas em resposta a alteração da disponibilidade hídrica do solo.

\section{MATERIAL E MÉTODOS}

O experimento foi conduzido no período de dezembro de 2012 a maio de 2013 na Escola Superior de Agricultura "Luiz de Queiroz” (ESALQ-USP), localizada no município de Piracicaba-SP, com coordenadas geográficas locais de $22^{\circ} 43^{\prime \prime}$ de latitude sul e $47^{\circ} 38^{\prime \prime}$ de longitude oeste, com altitude de aproximadamente 547 metros. O clima do município é classificado como subtropical úmido com estiagem no verão.

Foram utilizados 10 genótipos de arroz, três pertencentes ao grupo moderno (BRS-Cirrad, BRS-Curinga e BRSPrimavera) e sete ao grupo tradicional (Douradão, Agulha Ligeiro, Bolinha, Batatais, Três Meses Branco, Beira Campo e De Morro), cultivados em vasos e submetidos a duas condições de disponibilidade hídrica do solo: manutenção da umidade do solo próximo à capacidade máxima de sua retenção de água e manutenção da umidade do solo equivalente a uma tensão de $40 \pm 2 \mathrm{kPa}$.

A semeadura foi realizada em bandejas plásticas de $50 \times 35 \times 10 \mathrm{~cm}$. Cada bandeja foi preenchida com $15 \mathrm{~kg}$ de areia lavada utilizada como substrato. Quando as plantas apresentaram a segunda folha expandida, estádio vegetativo V2, foi realizado o transplante de três mudas por vaso. Os vasos apresentavam volume de 4 litros, e continham $3,6 \mathrm{~kg}$ solo adubado como substrato. Quando as plantas apresentaram a quarta folha expandida (estádio V4) realizouse o desbaste, deixando-se apenas uma planta por vaso. A adubação foi realizada no 


\section{ASSIMILAÇÃO DE CO2 DE GENÓTIPOS MODERNOS E TRADICIONAIS DE ARROZ SUBMETIDOS AO ESTRESSE HÍDRICO}

momento do plantio de acordo com a análise química do solo (Tabela 1), utilizando-se as doses de 6 g e 3 g dos fertilizantes NPK (414-8) e Yoorin Master por vaso, correspondendo às doses de 1250 e $600 \mathrm{~kg} \mathrm{ha}^{-}$ 1, respectivamente. $\mathrm{Na}$ adubação de cobertura, aplicaram-se a cada dois dias, 90 $\mathrm{mL}$ de solução nutritiva contendo as seguintes concentrações dos fertilizantes: 615 $\mathrm{mgL}^{-1}$ de $\mathrm{Ca}\left(\mathrm{NO}_{3}\right)_{2} ; 208 \mathrm{mg} \mathrm{L}^{-1}$ de $\mathrm{KNO}_{3}$; $179 \mathrm{mg} \mathrm{L}^{-1}$ de MKP; $176 \mathrm{mg}$ de $\mathrm{K}_{2} \mathrm{SO}_{4} ; 444$ $\mathrm{mg} \mathrm{L}^{-1}$ de $\mathrm{MgSO}_{4}$ e $50 \mathrm{mg} \mathrm{L}^{-1}$ de Conmicros como fonte de micronutrientes.

Durante o período vegetativo (estádio V1 até Vn, que corresponde à formação do colar da primeira folha até a formação do colar da $n$ folha, folha bandeira) e inicio da fase reprodutiva (estádio $\mathrm{R} 0$ a $\mathrm{R} 2$, que corresponde à iniciação da panícula até a elongação da panícula) as plantas foram conduzidas dentro de casa vegetação e os vasos mantidos sob condição hídrica próxima à capacidade máxima de retenção de água do solo. No período reprodutivo (do estádio R3, que corresponde saída da panícula, até o estádio R6, início do enchimento do grão),as plantas foram conduzidas dentro de câmara de crescimento (Fitotron), onde foram aplicados os tratamentos com maior e menor disponibilidade hídrica. Um dia antes da entrada dos vasos no Fitotron, os vasos do tratamento com menor disponibilidade hídrica não receberam irrigação e tiveram sua tensão de água do solo monitorada a cada 30 minutos por um tensímetro digital (Bringer Pressure Gauge), inserido em um tensiômetro instalado em cada vaso a $10 \mathrm{~cm}$ de profundidade, até atingir-se uma tensão da água do solo de $40 \pm 2 \mathrm{kPa}$. Após a tensão ser atingida, os tensiômetros eram retirados e os vasos pesados em balança digital, determinando-se assim o peso equivalente a umidade do solo na referida tensão. Os vasos que não receberam tratamentos com menor disponibilidade hídrica foram irrigados de maneira a manter o solo próximo à sua capacidade máxima de retenção.

Tabela 1. Análise química do solo no qual as plantas foram cultivadas, antes das adubações

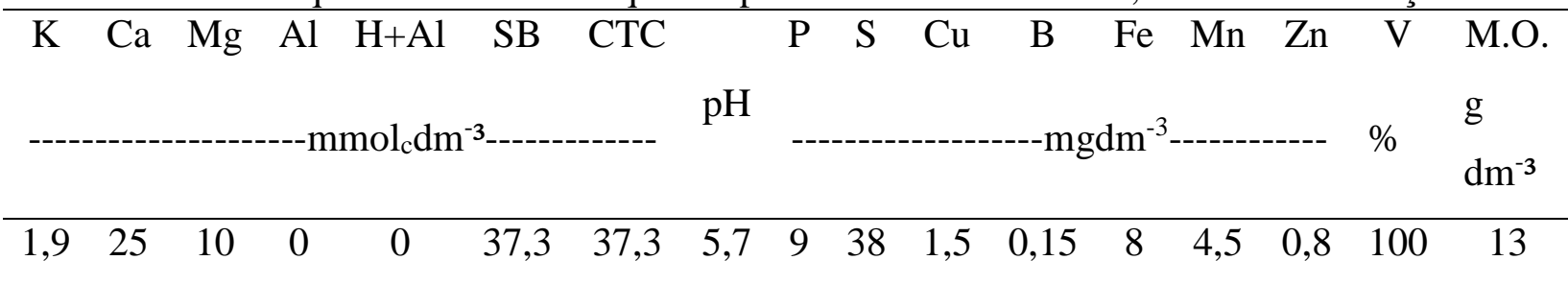


Após a pesagem, os vasos de ambos os tratamentos foram colocados dentro de uma câmara de crescimento (FITOTRON: modelo E15, Conviron, Winnipeg, Canadá) com condições climáticas controladas (fotoperíodo de 12 horas, das 06:00 às 18:00 h), intensidade luminosa entre $400 \sim 500$ $\mu$ mol de quanta $\mathrm{m}^{-2} \mathrm{~s}^{-1}$, umidade relativa do ar de $50 \% \pm 3 \%$, e temperatura do ar de $25^{\circ} \mathrm{C} \pm$ $1^{\circ} \mathrm{C}$. onde permaneceram por um período de aclimatação de cinco dias antes das avaliações. Os vasos com menor disponibilidade hídrica foram irrigados quatro vezes ao dia, aplicando-se a quantidade de água necessária pra se reestabelecer seu peso ao valor determinado na pesagem $(40 \pm 2 \mathrm{kPa})$. Os vasos com maior disponibilidade foram irrigados com quantidades de água necessárias para manter a umidade do solo sempre próxima à capacidade de retenção.

As leituras de assimilação $(A)$ foram realizadas no terço central da folha bandeira do colmo principal, dentro do Fitotron, entre 9:00 e 11:00 horas da manhã, com o uso de um analisador de gases por infravermelho (Infrared Gas Analyzer - IRGA, modelo LI6400 da Li-Cor, Lincoln, NE, USA.). O equipamento foi regulado para utilizar concentrações de $400 \mathrm{~mol} \mathrm{~mol}^{-1} \mathrm{CO}_{2}$ no ar da referência utilizado na câmara de fotossíntese do IRGA. A densidade de fluxo de fótons fotossinteticamente ativos $(D F F F A)$ foi de $1000 \mu \mathrm{mol}$ [quanta] $\mathrm{m}^{-2} \mathrm{~s}^{-1}$. O tempo mínimo de equilíbrio estabelecido para as leituras foi de 5 minutos, quando o Coeficiente de Variação do equipamento (CV) encontravase abaixo de $1 \%(\mathrm{CV}<1 \%)$.

O delineamento experimental utilizado foi de blocos casualizados, com quatro repetições e vinte tratamentos arranjados em um esquema fatorial $10 \times 2$ (10 genótipos e 2 condições de disponibilidade hídrica), totalizando 80 unidades amostrais. Cada unidade experimental foi constituída por uma planta por vaso, no qual, realizaramse três leituras. Os resultados obtidos foram submetidos à análise de variância (ANOVA) e as médias comparadas entre si pelo teste de Tukey a 5\% de probabilidade.

\section{RESULTADOS E DISCUSSÃO}

A análise de variância (Tabela 2) demonstra a existência de diferenças significativas $(\mathrm{P} \leq 0,01)$ entre os genótipos, condições hídricas e a interação genótipo x condições hídricas. 
Tabela 2. Resumo da análise da variância para a Taxa de assimilação de $\mathrm{CO}_{2}\left(A ; \mu \mathrm{mol}\left[\mathrm{CO}_{2}\right]\right.$ $\left.\mathrm{m}^{-2} \mathrm{~s}^{-1}\right)$ dos genótipos $(\mathrm{G})$ de arroz em relação às condições hídricas $(\mathrm{E})$

Causas da variação

GL

Quadrado Médio

\begin{tabular}{lcc}
\hline \multirow{2}{*}{ Causas da variação } & GL & Quadrado Médio \\
\cline { 3 - 3 } & & $A^{(1)}$ \\
\hline Genótipo(G) & 9 & $0,802^{* *}$ \\
Condições Hídricas (E) & 1 & $14,251^{* *}$ \\
G x E & 9 & $0,520 * *$ \\
Resíduo & 217 & 0,061 \\
Média & - & 10,67 \\
CV exp $(\%)$ & - & 7,48
\end{tabular}

** valor significativo ao nível de $1 \%$ de probabilidade de erro pelo teste $\mathrm{F} .{ }^{(1)}$ dados transformados por $(n+0,5)^{0,5}$ pelo teste de Hartley ao nível de $5 \%$ de probabilidade de erro. $n=$ dado amostrado. GL $=$ graus de liberdade, $\mathrm{CV}_{\exp }=$ coeficiente de variação experimental

$\mathrm{Na}$ condição de menor disponibilidade hídrica, os valores de assimilação de $\mathrm{CO}_{2}$ variaram entre 6,8 10 $\mu \mathrm{mol}\left[\mathrm{CO}_{2}\right] \mathrm{m}^{-2} \mathrm{~s}^{-1}$ (Tabela 3$)$. Tais valores estão dentro da variação observada por Centritto et al. (2009), para genótipos de arroz de terras altas e irrigado submetidos à condições de défict hídrico moderado e severo.Nessa condição, os genótipos tradicionais Douradãoe Batatais apresentaram os maiores valores em relação aos demais. Diferenças significativas da taxa de assimilação de $\mathrm{CO}_{2}$ entre genótipos de arroz submetidos a condições hídricas limitantes também foram relatadas por (YOSHIDA, 1975; DINGKUHN et al., 1989; DINGKUHN, 1991; JODO, 1995; LAFITE et al., 2006; CENTRITTO et al., 2009).No entanto, percebe-se que a variação entre os valores superiores (Douradãoe Batatais) e os inferiores (Agulha Ligeiro e BRSPrimavera)foram de apenas $28 \%$, sendo essa variação inferior às observadas porCentrittoet al. (2009) e por Lafitteet al. (2006), que foram da ordemde $62 \%$ e $75 \%$, respectivamente. Os demais genótipos apresentaram valores semelhantes (Tabela 3).Em geral, constata-se que os genótipos apresentaram comportamento fotossintético semelhante, como relatado por Turner et al. (1986), que avaliaram sete genótipos de arroz em sistema de sequeiro e por Machado et al. (1996), que avaliaram dois cultivares de arroz de terras altas em tratamentos com e sem dificiência hídrica. 
Tabela 3. Valores médios da taxa de assimilação líquida de $\mathrm{CO}_{2}\left(A ; \mu \mathrm{mol}\left[\mathrm{CO}_{2}\right] \mathrm{m}^{-2} \mathrm{~s}^{-1}\right) \mathrm{e}$ desvio padrão $(D P)$ dos genótipos de arroz de terras altas, em relação a condição de disponibilidade hídrica:(i) $40 \mathrm{kPa}$ de tensão e (ii) próximo à capacidade máxima de retenção de água no solo do vaso $(P C M R)$

\begin{tabular}{ccccccc}
\hline \multirow{2}{*}{ Genótipos } & \multicolumn{7}{c}{ Condições Hídricas } \\
\cline { 2 - 7 } & $(A)$ & $D P$ & & $(A)$ & $D P$ \\
\hline Três Meses Branco & 9,37 & $\pm 2,0$ & $\mathrm{ABb}$ & 11,4 & $\pm 0,6$ & $\mathrm{CDEa}$ \\
Agulha Ligeiro & 7,57 & $\pm 0,2$ & $\mathrm{BCb}$ & 11,8 & $\pm 2,7$ & $\mathrm{CDEa}$ \\
Batatais & 9,94 & $\pm 1,7$ & $\mathrm{Ab}$ & 11,31 & $\pm 0,6$ & $\mathrm{DEa}$ \\
Beira Campo & 9,68 & $\pm 1,9$ & $\mathrm{ABa}$ & 10,7 & $\pm 3,0$ & $\mathrm{CDa}$ \\
Bolinha & 9,25 & $\pm 2,1$ & $\mathrm{ABb}$ & 11,4 & $\pm 0,6$ & $\mathrm{CDEa}$ \\
BRS Cirrad & 9,7 & $\pm 1,1$ & $\mathrm{ABb}$ & 16,88 & $\pm 2,1$ & $\mathrm{Aa}$ \\
BRS Curinga & 9,79 & $\pm 2,2$ & $\mathrm{ABb}$ & 14,28 & $\pm 1,0$ & $\mathrm{ABa}$ \\
De Morro & 8,21 & $\pm 0,8$ & $\mathrm{ABCb}$ & 9,83 & $\pm 1,8$ & $\mathrm{Ea}$ \\
Douradão & 10,04 & $\pm 1,0$ & $\mathrm{Ab}$ & 12,82 & $\pm 1,1$ & $\mathrm{BCa}$ \\
BRS Primavera & 6,8 & $\pm 1,4$ & $\mathrm{Cb}$ & 12,62 & $\pm 1,8$ & $\mathrm{BCDa}$ \\
\hline
\end{tabular}

Nas colunas, médias seguidas pela mesma letra maiúscula, e nas linhas, médias seguidas por mesma letra minúscula não diferem significativamente pelo teste de Tukey ao nível de 5\% de probabilidade de erro

Na condição de maior disponibilidade hídrica, próximo à capacidade máxima de retenção de água pelo solo do vaso, o genótipo moderno BRS-Cirrad apresentou valores superiores em relação aos demais, seguido pelo BRS Curinga, que não diferiu estatisticamente dos genótipos BRSPrimavera e Douradão (Tabela 3).

Observa-se que, os genótipos modernos apresentam taxas fotossintéticas superiores aos tradicionais (Tabela 3). A superioridade na taxa de assimilação evidencia o motivo pelo qual os cultivares modernos, quando cultivados em condição de boa disponibilidade hídrica apresentam maiores produtividades em relação aos tradicionais (CEPEA, 2002; PINHEIRO, 2003). Pois, de acordo com Yoshida (1975) e Murchieet al. (1999), a maior parte do carbono armazenado nos grãos de arroz na fase de enchimento de grão são originados pela assimilação de $\mathrm{CO}_{2}$ da folha bandeira, a qual, neste estudo, foi a folha utilizada para as leituras.

Os valores de assimilação de $\mathrm{CO}_{2}$, para ambos os genótipos variaram entre 
9,83 16,88 $\mu \mathrm{mol}\left[\mathrm{CO}_{2}\right] \mathrm{m}^{-2} \mathrm{~s}^{-1}$ (Tabela 3).

Esses valores encontram-se abaixo dos descritos para outros genótipos de arroz irrigado e de sequeiro avaliados sob condições hídricas não limitantes (TURNER et al., 1986; MACHADO \& LAGÔA 1994; MACHADO et al., 1996; FALQUETO et al., 2007; MORADI \& SMAIL, 2007). No entanto, estão dentro da variação observada por Centrito et al. (2009) em genótipos de arroz de terras altas e irrigado mantios sob disponibilidade hídrica próxima à capacidade de campo.

As condições controladas de radiação luminosa (400 500 $\mu \mathrm{mol}$ [quanta] $\left.\mathrm{m}^{-2} \mathrm{~s}^{-1}\right)$ e de temperatura $\left(25^{\circ} \mathrm{C}\right)$ utilizadas dentro do Fitotron podem ter promovido as menores taxas de assimilação observadas nesse trabalho. Lorençoni et al. (2010) menciona que temperaturas na faixa de 28 a $32^{\circ} \mathrm{C}$ são ideais para o crescimento e desenvolvimento do arroz de terras altas. Além disso, Machado \& Lagoa (1994) e, Machado et al., (1996) relatam que a taxa de assimilação em arroz tende a aumentar quando as intensidades luminosas aumentam até 800 1200 $\mu \mathrm{mol}$ [quanta] $\mathrm{m}^{-2} \mathrm{~s}^{-1}$.

No entanto, de acordo com Hubbart et al. (2007), as cultivares modernas apresentam ampla adaptação às varições da disponibilidade de luz, apresentando capacidade fotossintética superior aos tradicionais, mesmo sob diferentes condições de disponibilidade de luz. Evidências semelhantes, também são descritas por Falqueto et al. (2007), que ao compararem a taxa fotossintética de dois genótipos de arroz irrigado com potenciais produtivos contrastantes (alto e baixo), atribuíram a diferença de produtividade entre os genótipos, a diferença na eficiência com a qual a energia luminosa é absorvida e convertida em carboidratos. Tal fato ajuda a explicar porque os maiores valores de assimilação apresentadados pelos genótipos modernos em relação aos tradicionais (Tabela 3), mesmo nas condições de disponibilidade de radiação luminosda e temperatura aqui utilizadas.

Independente da intensidade de radiação, percebe-se qiue as maiores taxas de assimilação dos genótipos modernos (BRSCirrad, BRS-Curinga e BRS-Primavera) foram determinadas em principio por sua maior capacidade fotossintética em relação aos tradicionais, o que também relatado por Kuroda \& Kumura (1990), Jiang et al.(2002), Zhang \& Kokubum (2004) e Sasaki \& Ishii (2004).

Ao se comparar os genótipos nas duas condições de disponibilidade hídrica, observa-se que todos os genótipos 
apresentaram reduções da taxa de assimilação de $\mathrm{CO}_{2}$, exceto o Beira Campo (Tabela 3). Reduções da assimilação de $\mathrm{CO}_{2}$ sob diminuição da disponibilidade hídrica tem sido amplamente relatada em arroz (TURNER, et al., 1986, KONDO et al., 2000) e outras culturas (WONG et al., 1979; TURNER \& BEGG, 1981; FARQRHAR \& SARKEY, 1982). No entanto, percebe-se que os genótipos modernos apresentaram os maiores percentuais médios de redução $(40,8 \%)$ em relação aos tradicionais $(18,8 \%)$. Esse comportamento indica a maior susceptibilidade dos genótipos modernos ao déficit hídrico.

Levando-se em conta que a taxa fotossintética da folha bandeira é a principal determinante da produtividade em arroz (MURCHIE et al, 1999), e que os fatores que reduzem a taxa de fotossíntese da folha bandeira durante o período reprodutivo podem limitar a produção de grãos (DINGKUHN et al., 1989), torna-se claro porque apesar do maior potencial produtivo, as variedades cultivadas modernas de arroz de terras altas apresentam baixos rendimentos sob déficit hídrico (CRUSCIOL et al., 1999; ARF et al., 2000; CRUSCIOL et al., 2003a,b).Esse comportamento parece estar relacionado com o processo de melhoramento pelo qual os genótipos modernos foram submetidos.

O objetivo principal dos programas de melhoramento que deram origem aos genótipos modernos de arroz de terras altas atualmente utilizados foi a obtenção de materiais com elevado potencial produtivo, sendo para tanto realizados sob condições hídricas não limitantes, de maneira a favorecer a expressão de todo potencial produtivo dos genótipos avaliados. No entanto, em contra partida, observa-se que os genótipos modernos apresentam elevadas reduções de produtividade sob condições hídricas limitantes, ou seja, são pouco tolerantes ao déficit hídrico.

No caso dos genótipos tradicionais, o processo de seleção empírico foi realizado baseando-se na escolha dos materiais mais adaptados às condições locais de cultivo. Levando-se em conta que os veranicos são fenômenos de ocorrência frequente em quase todas as regiões produtoras de arroz de terras altas, não é de se estranhar que os materiais tradicionalmente selecionados apresentem menor potencial produtivo mesmo sob condições de boa disponibilidade hídrica, e maior tolerância ao estresse hídrico (menores reduções de produtividade) quando submetidos a condições de déficit hídrico. 


\section{CONCLUSÕES}

Os genótipos tradicionais avaliados apresentaram-se mais tolerantes à seca quanto à taxa de assimilação de $\mathrm{CO}_{2}$. Esses genótipos podem ser selecionados para serem possíveis genitores em programas de melhoramento genético que objetivam desenvolver cultivares tolerantes à seca, embora apresentem menores valores de assimilação em condição sem déficit hídrico.

Em condição sem déficit hídrico, os genótipos modernos apresentam maiores taxas de assimilação de $\mathrm{CO}_{2}$, porém, quando submetidos à deficiência hídrica, apresentam as maiores reduções de assimilação, dessa forma, demonstrando maior suscetibilidade ao déficit hídrico.

\section{AGRADECIMENTOS}

Os autores agradecem a CAPES pela disponibilização de bolsa de estudos.

\section{REFERENCIAS BIBLIOGRAFICAS}

ADORIAN, G.C. Caracterização da diversidade fenotípica existente em uma coleção nuclear de arroz de terras altas. 2010. Dissertação (Mestrado em Produção Vegetal) - Universidade Federal do Tocantins/CAUG - Universidade Federal do Tocantins, Gurupi, 72p. 2010.
AREIAS, R.G. de B.M.; PAIVA, D.M. de; SOUZA, S.R.; FERNANDES, M.S. Similaridade genética de variedades crioulas de arroz, em função da morfologia, marcadores RAPD e acúmulo de proteína nos grãos. Bragantia, Campinas, v. 65, n. 1, p. 19-28. 2006.

ARF, O.; RODRIGUES, R.A.F.; SÁ, M.E.; CRUSCIOL, C.A.C. Influência da época de semeadura no comportamento de cultivares de arroz (Oryza sativa L.) irrigado por aspersão na região de Selvíria (MS). Pesquisa Agropecuária Brasileira, Brasília, v. 35, n. 10, p. 19671976, 2000.

CENTRITTO, M.; LAUTERI, M,; MONTEVERDI, M.C.; SERRAJ, R. Leaf gas exchange, carbon isotope discrimination, and grain yield in contrasting rice genotypes subjected to water deficits during the reproductive stage, Journal of Experimental Botany, Oxford, v. 60, p. 2325-2339, 2009.

CEPEA (CENTRO DE ESTUDOS AVANÇADOS EM ECONOMIA APLICADA). Projeto pioneiro pesquisa arroz de terras altas. 2002. Disponível em: <http://www.cepea.esalq.usp.br>. Acesso em: 14 abr. 2009.

CRUSCIOL, C.A.C.; ARF, O.; SORATTO, R.P.; MACHADO, J.R. Extração de 
macronutrientes pelo arroz de terras altas sob diferentes níveis de irrigação por aspersão e de adubação. Revista Brasileira de Agrociência, Pelotas, v. 9, n. 2, p. 145-150, 2003a.

CRUSCIOL, C.A.C.; ARF, O.; SORATTO, R.P.; MACHADO, J.R. Influência de lâminas de água e adubação mineral na nutrição e produtividade de arroz de terras altas. Revista Brasileira de Ciência do Solo, Viçosa, v. 27, n. 4, p. 647-654, 2003b.

CRUSCIOL, C.A.C.; MACHADO, J.R.; ARF, O.; RODRIGUES, R.A.F. Componentes de produção e produtividade de grãos de arroz de sequeiro em função do espaçamento e da densidade de semeadura, Scientia Agricola, Piracicaba, v. 56, n. 1, p. 53-62, 1999.

DINGKUHN, M.; DE DATTA S.K.; DORFFLING, K.; JAVELLANA, C. Varietal differences in leaf water potential, leaf net $\mathrm{CO}_{2}$ assimilation, conductivity, and water use efficiency in upland rice. Australian Journal of Agricultural Research, Victoria, v. 40, p. 1183-1192, 1989.

DINGKUHN, M.; FARQUHAR, G.D.; DE DATTA, S.K.; OTOOLE, J.C. Discrimination of ${ }^{13} \mathrm{C}$ among upland rices having different water use efficiencies.
Australian Journal of Agricultural

Research. Victoria, v. 42, p. 1123-1131, 1991.

FALQUETO, A.R.; CASSOL, D.; 2, MAGALHÃES JÚNIOR, A.M. DE.; OLIVEIRA, A.C.; BACARIN, M.A. Características fotossintéticas em Cultivares de Arroz com Produção Contrastante. Revista Brasileira de Biociências, Porto Alegre, v. 5, p. 582584, 2007.

FARQUHAR, G.D.; SHARKEY, T.S. Stomatal conductance and photosynthesis. Annual Review of Plant Physiology, Palo Alto, v. 33, p. 317-345, 1982.

HUBBART, S.; PENG, S.; HORTON, P.; CHEN, Y.; MURCHIE, E.H. Trends in leaf photosynthesis in historical rice varieties developed in the Philippines since 1966. Journal of Experimental Botany, Oxford, v. 58, p. 3429-2438, 2007.

JIANG, H.; WANG, X-H; DENG, O-Y; YUAN, L-P; XU, D-Q. Comparison of some photosynthetic characters between two hybrid rice combinations differing in yield potential. Photosynthetica. Prague, v. 40, p. 133-137, 2002.

JODO, S. Water use and drought resistance. In: MATSUO, T.; KUMAZAWA, K.; ISHII, R.; ISHIHARA, K.; HIRATA, H. 
(Ed.). Science of the rice plant physiology. Tokyo: Nosan Gyoson Bunda Kyokai, 1995. chap. 2, p. 461-483.

KUMAR, A.; BERNIER, J.; VERULKAR, S.; LAFITTE, H.R.; ATLIN, G.N. Breeding for drought tolerance: direct selection for yield, response to selection and use of drought-tolerant donors in upland and lowland-adapted populations.

Field Crops Research, Amsterdam, v. 107, p. 221-231, 2008.

KURODA, E.; KUMURA, A. Difference in single leaf photosynthesis between old and new rice varieties. I. Single-leaf photosynthesis and its dependence on stomatal conductance. Japanese Journal of Crop Science, Tokyo, v. 59, p. 283292, 1990.

LAFITTE, H.R.; LI, Z.K.; VIJAYAKUMA, C.H.M.; GAO, Y.M.; SHI, Y.; XU, J.L.; FU, B.Y.; YU, S.B.; ALI, A.J.; DOMINGO, J.; MAGHIRANG, R.; TORRES, R.; MACKILL, D. Improvement of rice drought tolerance through backcross breeding: Evaluation of donors and selection in drought nurseries.

Field Crops Research, Amsterdam, v. 97, p. 77-86, 2006.

MACHADO, E.C.; LAGÔA, A.M.M.A. Trocas gasosas e condutância estomática em três espécies de gramíneas. Bragantia, Campinas, v. 53, p. 141-149, 1994.

MACHADO, E.C.; LAGÔA， A.M.M.A.; AZZINI, L.E.; TISSELLI FILHO, O. Trocas gasosas e relações hídricas em dois cultivares de arroz de sequeiro submetidos à deficiência hídrica, em diferentes fases do crescimento reprodutivo. Revista Brasileira de Fisiologia Vegetal, Londrina, v. 8, p. 139-147, 1996.

MORADI, F.; ISMAIL, A.M. Responses of Photosynthesis, Chlorophyll Fluorescence and ROS-Scavenging Systems to Salt Stress During Seedling and Reproductive Stages in Rice. Annals of Botany, London, v. 99, p. 1161-1173, 2007.

MURCHIE, E.H.; CHEN, Y.Z.; HUBBART, S.; PENG, S.; HORTON, P. Interactions between senescence and leaf orientation determine in situ patterns of photosynthesis and photoinhibition in field-grown rice. Plant Physiology, Washington, v. 119, p. 553-563, 1999.

MURCHIE, E.H.; HORTON, P. Acclimation of photosynthesis to irradiance and spectral quality in British plant species: chlorophyll content, photosynthetic capacity and habitat preference. Plant, Celland Environment, Oxford, v. 20, p. 438-448, 1997. 
PINHEIRO, B.S. Cultivo do arroz de terras altas: Características da cultura. 2003. Embrapa Arroz e Feijão. Disponível em: $<$ http://sistemasdeproducao.cnptia.embra pa.br/FontesHTML/Arroz/ArrozTerrasAlt as/index.htm> Acesso em: 29 jul. 2012.

RABELlO, A.R.; RANGEL, P.H.N.; GUIMARÃES, C.M.; SALES, R.M.O.B.; SILVA, F.R. DA.; COSTA, M.M.C.; TOGAWA, R.C.; MÁRCIO, E.F.; MEHTA, A. Expressão diferencial em genótipos de Oryza sativa contrastantes para a tolerância à seca. Boletim de pesquisa e Desenvolvimento 156, Brasília, 2006, 21p.

RANGEL, P.H.N.; GUIMARÃES, E.P.; RABELO, R.R. Melhoramento genético do arroz irrigado no Nordeste do Brasil. 2011. Disponível em: <http://www.cpatsa.embrapa.br:8080/cata logo/livrorg/arrozirrigado.pdf $>$. Acesso em: 12 mai. 2011.

SASAKI, H.; ISHII, R. Cultivar differences in leaf photosynthesis of rice bred in Japan. Photosynthesis Research, Dordrecht, v.32, p. 139-146, 2004.

STONE, L.F.; PEREIRA, A.L. Sucessão arroz-feijão irrigado por aspersão: efeitos de espaçamento, entrelinhas, adubação e cultivar na produtividade e nutrição do arroz. Pesquisa Agropecuária
Brasileira, Brasília, v. 29, n. 11, p. 1701$1713,1994$.

TERRA, T.G.R. Avaliação de características morfofisiológicas de tolerância à seca em uma coleção nuclear de acessos de arroz de terras altas (Oryza sativa L.). 2008. 81 p. Dissertação (Mestrado em Produção Vegetal) - Universidade Federal do Tocantins/CAUG - Universidade Federal do Tocantins, Gurupi, 2008.

TURNER, N.C.; BEGG, J.E. Plant-water relations and adaptation to stress. Plant and Soil, The Hague, v. 58, p. 97-131, 1981.

TURNER, N.C.; OTOOLE, J.C.; CRUZ, R.T.; NAMUCO, O.S.; AHMAD, S. Responses of seven diverse rice cultivars to water deficits. 1. Stress development, canopy temperature, leaf rolling and growth. Field Crops Research, Amsterdam, v. 13, p. 257-271, 1986.

VENUPRASAD, R.; LAFITTE, H. R.; ATLIN, G.N. Response to direct selection for grain yield under drought stress in rice.Crop Science, Madison, v. 47, p. 285293, 2007.

WONG, S.C.; COWAN, I.R.; FARQUHAR, G.D. Stomatal conductance correlates with photosynthetic capacity. Nature, London, v. 282, p. 424-426, 1979. 
YOSHIDA, S. Factors that limit the growth and yield of upland rice. In: Major Research in Plant Rice. Los Baños, Philippines. International Rice Research Institute, p. 46-71, 1975.

ZHANG, W.H; KOKUBUM, M. Historical changes in grain yield and photosynthetic rate of rice cultivars released in the 20th Century in Tohoku Region. Plant Production Science. Tokyo, v. 7, p. 3644, 2004. 